\title{
Knowledge Network Internetworking
}

\author{
Kirstin Schwidrowski \\ Universität Siegen, Institut für Didaktik der Informatik und E-Learning \\ Hölderlinstr. 3, 57076 Siegen, Germany \\ schwidrowski@die.informatik.uni-siegen.de, \\ WWW home page: http://www.die.informatik.uni-siegen.de
}

\begin{abstract}
The convergence of informatics systems and media systems has caused a lack of educational concepts for long-term knowledge. We assume that gaining informatics competencies enables adults to meet the demands of today's society. Therefore, didactics of informatics has to contribute educational concepts. In this paper, we introduce a strategy to structure the domain Internetworking. Aim of our research is to develop a Didactic System for Internetworking. By applying the presented strategy a knowledge network is constructed. It represents knowledge with an educational value for handling informatics systems as well as various possibilities of sequencing this knowledge for learning processes. Thereby, findings in informatics and didactics of informatics are considered. The knowledge network is improved by results of a case study. Dimensions of knowledge networks are informatics principles, knowledge level, application, and matter.
\end{abstract}

\section{Media Upheaval and implications to Didactics of Informatics}

Informatics systems and media systems have converged. Therefore, media usage has changed. As a result a lack in educational frameworks has emerged. Therefore, didactics of informatics contribute concepts. Research objective is development of an e-learning framework [1]. Thereby, we concentrate on the domain Internetworking. We regard its structure, communication, and information security.

In this paper, we present a strategy to structure learning processes for acquiring informatics knowledge regarding Internetworking. Thereby, we consider adult learners with no prior knowledge informatics and heterogeneous experience in handling informatics systems. A knowledge network is the result of applying the strategy. The network represents a structure of a domain from a didactic point of view. Arrangement of knowledge objects provides various learning sequences. Findings in informatics, didactic of informatics, and adult learning are considered to deduce sequence of learning matter. 


\section{Approaches for Structuring Learning Processes and Domain}

Brinda and Schubert have introduced a Didactic System [2]. This educational framework combines a knowledge network, exercise classes, and exploration modules. We follow up this approach to deduce a Didactic System for Internetworking. Stechert has applied a learner-centred cognitive approach for improving informatics system understanding [3]. Observable behavior, internal structure, and implementation are characteristics of informatics systems. These characteristics structure the learning matter. Voß has proposed an educational concept for using standard software [4]. She has addressed adult learners. Her approach offers a cognitive access based on informatics modelling. Learners get to know the internal structure of a text or table document. This knowledge is combined with applying standard software. In our assumption, informatics knowledge enables to analyze, evaluate, and reflect functioning of informatics systems. In particular, learners know structure of Internetbased media systems, states of media processes, and the combination of both.

\section{Learning Barriers}

The design of an educational framework includes structuring of learning matter. At first, informatics principles with an educational value have been identified. Therefore, we have evaluated their relevance for Internet structure and processes. Two additional criteria have been generality and appropriate complexity. Identified informatics principles have been protocols, client-server model, and IP-addressing. Integrity, availability, and confidentiality are introduced as quality factors of an informatics system. During a case study several problems of using the Internet have been identified [1]. The result is summarized in Tab. 1. We have used it for deducing a strategy to structure the domain Internetworking.

\section{Problem}

$\mathrm{P}_{1} \quad$ Domain specific terminology (e.g. client, server, html) is not understandable.

$\mathrm{P}_{2} \quad$ Basic IT-knowledge like working with common data formats is not known.

$\mathrm{P}_{3} \quad$ Explanations that are based on experience lack in integrity.

$\mathrm{P}_{4}$ Informatics knowledge is restricted to specific software.

$\mathrm{P}_{5}$ Personal benefit of learning informatics is not obvious.

$\mathrm{P}_{6} \quad$ No alternative learning matters are offered.

$\mathrm{P}_{7}$ Learning objectives are not useful for ordinary computer users.

Table 1: Problems of using the Internet

\section{Strategy for a Multi-Dimensional Knowledge Network}

A knowledge network offers information about the intended learning outcome and the relation of knowledge within a domain. Criteria for generating a knowledge network are deduced and listed in Tab. 2. 


\begin{tabular}{lll}
\hline & Requirement & Related Problems \\
\hline \hline $\mathrm{R}_{1}$ & Modeling alternative sequences of knowledge objects & $\mathrm{P}_{6}, \mathrm{P}_{7}$ \\
\hline $\mathrm{R}_{2}$ & Modeling optional knowledge objects & $\mathrm{P}_{7}$ \\
\hline $\mathrm{R}_{3}$ & Modeling knowledge objects according to their intensity & $\mathrm{P}_{1}, \mathrm{P}_{2}, \mathrm{P}_{3}$ \\
\hline $\mathrm{R}_{4}$ & Defining prior knowledge & $\mathrm{P}_{1}, \mathrm{P}_{2}, \mathrm{P}_{3}$ \\
\hline $\mathrm{R}_{5}$ & Using prior experience more obvious & $\mathrm{P}_{3}, \mathrm{P}_{4}, \mathrm{P}_{5}, \mathrm{P}_{6}$ \\
\hline $\mathrm{R}_{6}$ & Reflecting individual experiences and learning goals & $\mathrm{P}_{6}, \mathrm{P}_{7}$ \\
\hline
\end{tabular}

Table 2: Requirements for refining knowledge network structure

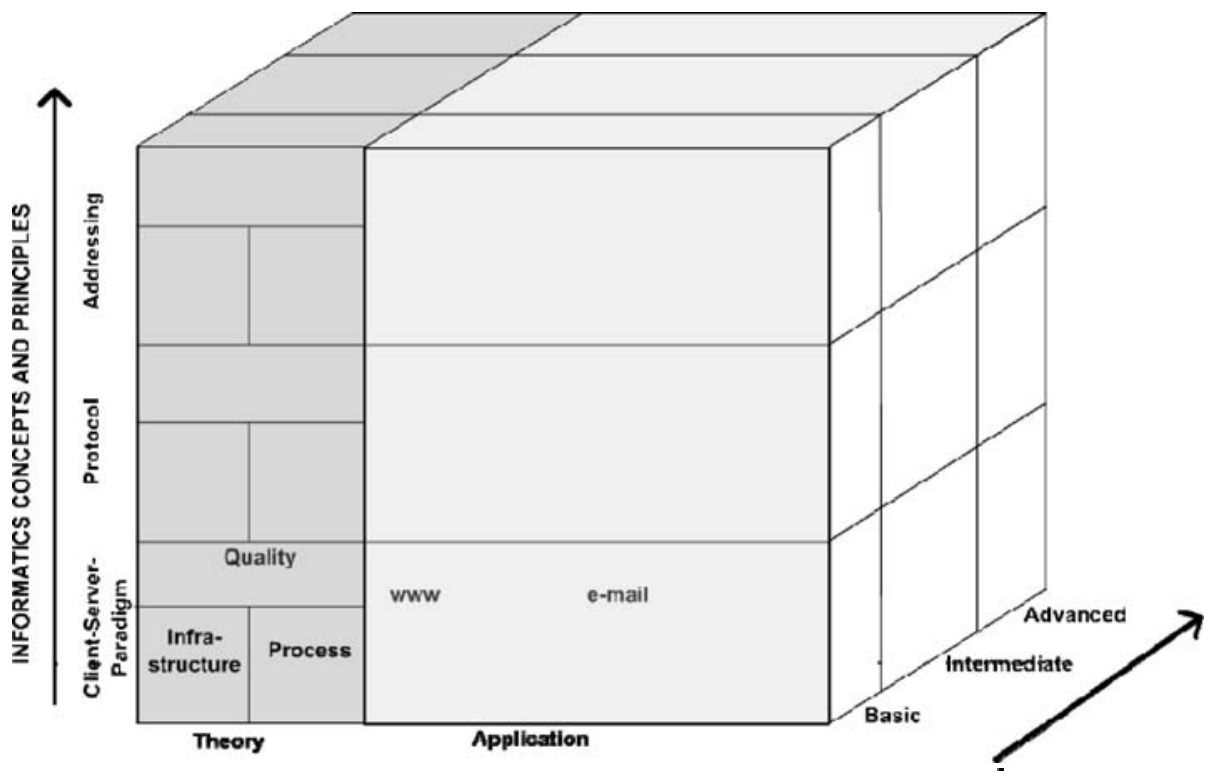

Fig 1. Multi-dimensional knowledge network 'internetworking'

We address elements of a knowledge network as knowledge objects. A knowledge object is a combination of an intended learning outcome and a domain specific location. The location is determined by dimensions informatics principle (IP), knowledge level (KL), application (A), and matter (M). Dimension IP explains basic structures and processes of the Internet. Dimension KL deals with complexity (see $\left.\mathrm{R}_{3}\right)$. Three levels are suggested: basic, intermediate, and advanced. The basic level emphasizes on terminology since this is a precondition for understanding (see $\left.\mathrm{R}_{4}\right)$. The intermediate level addresses handling with informatics systems under regular circumstances. The advanced level offers additional, not mandatory knowledge (see $\mathrm{R}_{2}$, $\mathrm{R}_{6}$ ). The dimension A assigns either a certain application (e.g. World Wide Web, email, groupware) or theory to every knowledge object. Thereby, theoretical aspect offers an abstraction of concrete applications of an informatics principle. It helps to transfer knowledge. The practical aspect exemplifies the theoretical knowledge by real life applications. This supports goal-oriented learning ( $\mathrm{see} \mathrm{R}_{6}$ ). The dimension matter reflects the problem that informatics systems are very complex such that is 
very difficult to analyze and evaluate them. Every informatics system has a structure. That is a static perspective. An informatics system is included in processes. This is a dynamic perspective. At least informatics systems are used in a specific context. According to it, they can be evaluated. Therefore, quality factors or preconditions have to be known.

A sequence of knowledge objectives is defined by the following rules. Regarding dimension IP, the default sequence is (1) client-server model, (2) protocols, and (3) addressing. Dimension KL has a fixed order. If a learner has already reaches a knowledge objective of basic or intermediate level, it can be omitted. All advanced knowledge objects are optional. A learner can decide whether to start with a theoretical or a practical knowledge object (see $\mathrm{R}_{5}$ ) at dimension A. But they are mandatory. The sequence of knowledge objects in dimension $\mathrm{M}$ is partly given. Before handling a knowledge object of the quality perspective the ones of the structure and the process have to be handled. In Fig. 1, the knowledge network is visualized by a cube. The initial points of the knowledge network are on the lower, front side. Learners cross this cube from there to the backside and then up to next stage of dimension IP.

\section{Remarks on Further Work}

In this paper, we have presented a strategy to deduce a knowledge network. It puts the emphasis on structuring the domain Internetworking. In the next step, the concept of knowledge networks will be refined. The current concept either results in a representation of a specific learning process or in a large, confusing one. Therefore, we will introduce macro and micro knowledge networks that address learning outcomes at the level of learning objectives and at competence level.

\section{References}

[1] K. Schwidrowski, Introducing Internetworking in Vocational Training, in: Proceeding of the IFIP Joint Working Conference iTET2007 "Information Technologies for Education and Training 2007", edited by C. Abbott and Z. Lustigova (ETIC, Prague, 2007), pp.154 - 161.

[2] T. Brinda and S. Schubert, Didactic System for object-oriented modeling, in: Proceedings of networking the learner/Computers in education, edited by D. Watson and J. Andersen (Kluwer, Boston, 2002), pp. 473 - 482.

[3] P. Stechert, Informatics system comprehension: A learner-centered cognitive approach to networked thinking, in: Education and Information Technologies 11(3) "Imagining the future for ICT and education", edited by B. Munro and D. Watson (Springer Netherlands, Dordrecht, 2006), pp. 305 - 318.

[4] S. Voß, Informatic Models in Vocational Training for Teaching Standard Software, in: Proceedings of ISSEP 2005 „Informatics for Secondary Schools - Evolution and Perspectives: From Computer Literacy to Informatics Fundamentals“, edited by R. Mittermeir, (Klagenfurt, 2005), pp. 145 - 155. 